\title{
Strongly bound excitons in metal-organic framework MOF-5: a many-body perturbation theory study \\ Aseem Rajan Kshirsagar, ${ }^{\dagger}$ Xavier Blase ${ }^{\ddagger}$ Claudio Attaccalite, ${ }^{\top}$ and Roberta Poloni ${ }^{*} \dagger$ $\dagger$ Univ. Grenoble Alpes, CNRS, Grenoble-INP, SIMaP, 38000 Grenoble, France \\ $\ddagger$ Univ. Grenoble Alpes, CNRS, Institut Néel, 38042 Grenoble, France \\ ๆ CNRS/Aix-Marseille Université, Centre Interdisciplinaire de Nanoscience de Marseille UMR 7325, Campus de Luminy, 13288 Marseille Cedex 9, France \\ Received February 4, 2021; E-mail: roberta.poloni@grenoble-inp.fr
}

\begin{abstract}
During the past years, one of the most iconic metalorganic frameworks (MOFs), MOF-5, has been characterized as a semiconductor by theory and experiments. Here we employ the $G W$ many-body perturbation theory in conjunction with the Bethe-Salpeter equation (BSE) to compute the electronic structure and optical properties of this MOF. The $G W$ calculations show that MOF-5 is a wide band-gap insulator with a fundamental gap of $\sim 8 \mathrm{eV}$. The strong excitonic effects, arising from highly localized states and low screening, result in an optical gap of $4.5 \mathrm{eV}$ and in an optical absorption spectrum in excellent agreement with experiments. The origin of the incorrect conclusion reported by past studies and the implication of this result are also discussed.
\end{abstract}

\section{Introduction}

Owing to their porous structure, chemical tunability and diversity, metal-organic frameworks (MOFs) are being actively studied for a wide range of applications. ${ }^{1-6}$ One of the first synthesized MOFs to exhibit relatively large porosity and chemical and thermal stability is MOF-5. ${ }^{7}$ This framework, made of $\mathrm{Zn}_{4} \mathrm{O}$ subunits connected by ligands of terephthalic acid, is probably the most iconic MOF and among the most studied ones. ${ }^{8}$ Since the early 2000 's, it has been the subject of a large number of studies reporting its chemical and physical properties, and assessing its applications in the fields of optoelectronics, ${ }^{9,10}$ gas separation, ${ }^{11,12}$ gas sensing, ${ }^{13}$ and catalysis. ${ }^{14-16}$

During these years, there have been several experimental studies reporting significant discrepancies in the description of the electronic structure, the optical excitations and photoluminescence in MOF-5. At first, it was identified as a semiconductor with an optical band gap of 3.4$3.7 \mathrm{eV}$ featuring a ligand to metal charge transfer excitation. ${ }^{10,17,18}$ Later, by using the photoluminescence spectra of MOF-5 for assessment of its purity, it was shown that the method of synthesis used by early studies leads to presence of $\mathrm{ZnO}$ impurities within the framework, and these impurities can exhibit the observed semiconductor behaviour. ${ }^{19}$ Subsequent experimental studies also showed that the photoluminescence and absorption spectra of MOF5 are very sensitive to the presence of water and structural damage, ${ }^{16,20,21}$ further raising questions on the early works. The studies that followed showed that the onset of the optical absorption of possibly impurity-free MOF-5 is located at $\sim 3.8-4.1 \mathrm{eV} .{ }^{16,20-25}$ In parallel, several computational works ${ }^{25-29}$ have established a semiconducting behav- ior of this MOF upon a computed band gap of around 3.6 eV using Kohn-Sham density-functional theory (DFT) with semi-local Perdew-Burke-Ernzerhof (PBE) parametrization of exchange-correlation (XC). In these studies, the accuracy of Kohn-Sham PBE to predict the electronic structure of MOF-5 was claimed on the basis of the good comparison with experimental optical gap. These considerations, however, are confusing and misleading for two reasons. First, the use of optical measurements to establish whether a material is a semiconductor or an insulator is questionable, because if strongly bound excitons are created the optical gap may be up to several $\mathrm{eV}$ smaller than the (fundamental) band gap. For this, photoemission spectroscopy should be employed to measure the fundamental gap which is defined as the difference between the ionization energy and the electron affinity. Second, the Kohn-Sham gap is not the fundamental gap, not even in exact Kohn-Sham theory ${ }^{30}$ and approximated functionals such as PBE are well known to give electronic band gaps almost $50 \%$ smaller than the fundamental gap. Besides, a small semiconducting gap is rather uncommon in MOFs, ${ }^{31,32}$ especially in those with highly-localized dispersionless bands such as in MOF- $5^{28}$ and it is not surprising that the claimed semiconducting behaviour may be viewed with skepticism. ${ }^{19,31,33}$

In this study, we employ ab initio many-body perturbation theory methods, such as the $G W$ approximation, ${ }^{34}$ together with the Bethe-Salpeter equation (BSE) ${ }^{35,36}$ formalism to compute the quasiparticle (photoemission) band gap and the two-body electron-hole bound states associated with neutral excitations to calculate the optical spectra of MOF5 and its ligand. These methods, originally implemented to study excited states in semiconductors, ${ }^{36-38}$ are recently gaining much momentum in the physical chemistry community. ${ }^{39-41}$ The $G W$ method yields the quasiparticle eigenvalues corresponding to the electron addition and removal energies and it allows for a quantitatively good estimate of the fundamental photoemission band gap. ${ }^{39,42}$

Our results show that MOF-5 is a wide-gap insulator with a quasiparticle $G W$ gap of $\sim 8 \mathrm{eV}$. The BSE yields an optical gap of $4.5 \mathrm{eV}$ with an intense absorption peak at $5.3 \mathrm{eV}$ in good agreement with experimental studies. ${ }^{24,43}$ The optical absorption is dominated by ligand-centered transitions at least up to $\sim 6 \mathrm{eV}$, also in agreement with inferences drawn in recent experimental studies. ${ }^{16,20}$ The large difference between the fundamental gap and optical band gap points to strongly bound excitons in the studied spectral region with binding energies of several eVs, previously not discussed in literature. 


\section{Methods}

The $G W$ quasiparticle eigenvalues are corrected perturbatively by replacing the exchange-correlation contribution to the KS eigenvalues by the $\Sigma\left(r, r^{\prime}, E\right)$ self-energy (computed within the $G W$ approximation), which accounts for the many-body exchange-correlation effects. An eigenvalues self-consistent $G W$ procedure is adopted to correct the DFT eigenvalues. ${ }^{44,45}$ In the first $G W$ iteration the KS eigenvalues and wavefunctions are used to compute the expectation value of the self-energy. Several $G W$ iterations are then performed by replacing the $\mathrm{KS}$ eigenvalues by the newest $G W$ quasiparticle values while keeping the KS eigenvectors frozen. Such procedure is repeated until the $G W$ gap is converged within $0.1 \mathrm{eV}$ to yield the so-called $e v G W$ quasiparticle energies. In what follows the $G W$ values always refer to evGW. The quasiparticle energies and the KS eigenvectors are then used to build the BSE electron-hole Hamiltonian. See the supporting information (SI) for more details. We perform BSE/ $G W$ calculations for MOF-5 using both periodic boundary conditions and molecular fragment models. Additionally, we compute the ligand (in solution) employed in the synthesis of MOF-5 for which several experimenal studies are available.

Periodic calculations The calculations of MOF-5 using periodic boundary conditions are performed using QUANTuM ESPRESSO ${ }^{46}$ for DFT and YAMBO ${ }^{47}$ for the $G W$ and BSE. Optimized norm-conserving SG15 pseudopotentials are used to model the interaction between ionic core and the valence electrons. ${ }^{48,49}$ For comparison, the $\mathrm{BSE} / G W$ calculations are performed using both semilocal PBE and the hybrid Gau-PBE which incorporates $25 \%$ of exact exchange at short range with Gaussian attenuation. ${ }^{50,51}$ See SI for the more details of the $G W$ and BSE calculations.

Fragment calculations For the calculations of the MOF-5 fragments and the MOF ligand we employ $\mathrm{NWCHEM}^{52}$ and ORCA ${ }^{53}$ for DFT, and FIESTA ${ }^{44,54}$ for BSE and $G W$. The DFT calculations are performed using PBE and PBE0. The conductor-like polarizable continuum model as implemented in ORCA is employed to account for solvation effects of the ligand in the ground state. At the GW and BSE level, a discrete polarizable model is used to calculate a reaction field matrix which effectively captures the dielectric screening of electronic excitations by the solvent (for the ligand) or the MOF environment (for the MOF fragments). ${ }^{55-57}$ The reaction field matrix is incorporated in the screened Coulomb potential $W$ used in $G W$ and BSE. This method has allowed us to correctly describe the electronic structure and the optical excitations of both solvated molecules, ${ }^{58}$ including azobenzene derivatives, ${ }^{59}$ and molecular crystals. ${ }^{56}$ See SI for more details.

\section{Results and discussion}

The experimental absorption spectrum of the BDC $(1,4 \mathrm{ben}-$ zenedicarboxylic) ligand measured in water ${ }^{60}$ is shown in Fig. 1. The BSE $/ G W / \mathrm{PBE} 0$ spectrum of BDC is reported in the same figure: it is computed using an embedding scheme which uses an effective medium with the total (ionic and electronic) and optical (electronic) dielectric response of water, for the ground state and excited states, respectively. See SI for more details. The BSE correctly reproduces all the spectral features reported experimentally. The first weak ex-

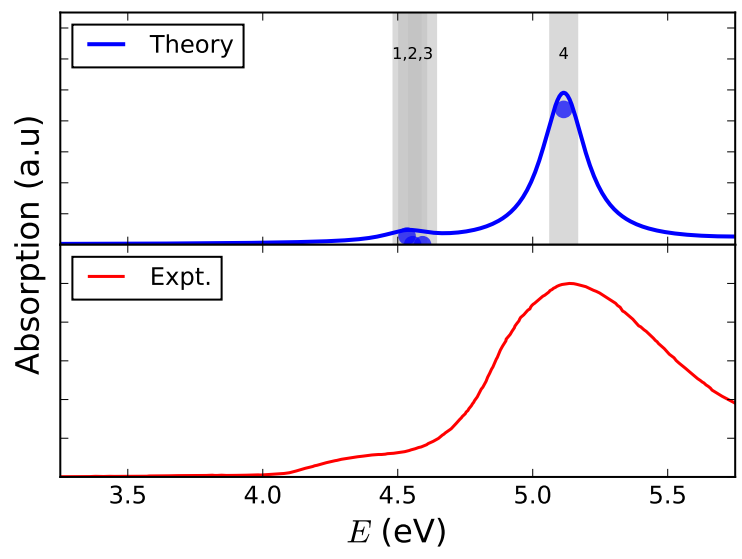

(a)

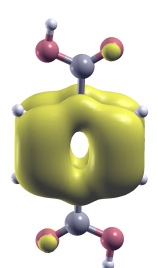

Hole 1

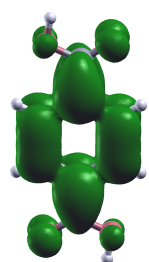

Electron 1

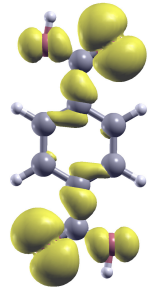

Hole 2

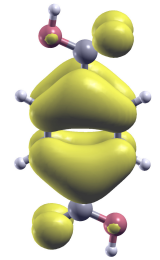

Hole 4

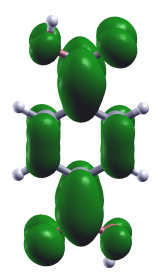

Electron 2

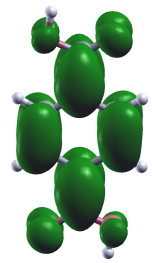

Electron 4
Figure 1. Comparison between the computed BSE/ $G W / \mathrm{PBE} 0$ spectrum and the experimental one from Ref. 60, for the BDC ligand (see text). The BSE spectrum is plotted by applying a Lorentzian broadening of $0.1 \mathrm{eV}$ (upper figure). Hole-averaged electron density and electron-averaged hole density plotted for the first four excitations (lower figure).

citation corresponds to the HOMO-LUMO transition with $\pi \rightarrow \pi^{*}$ character; it is mostly localized on the benzene ring and only marginally on the $\mathrm{O}$ atoms of the carboxylic group. This is followed by two dark excitons with zero oscillator strength which are charge transfer transitions from the $\mathrm{O}$ atoms to the benzene ring. The most intense excitation corresponds to another $\pi \rightarrow \pi^{*}$ transition of the benzene ring with small contributions of the $\mathrm{O}$ atoms. For a visual inspection of these excitations we plot the hole-averaged electron density and the electron-averaged hole density (see SI for more details) as obtained from BSE eigenvectors (lower panel of Fig. 1). A qualitatively similar result for both $G W$ and the BSE is predicted when using different starting point $\mathrm{XC}$ functionals such as PBE $0^{61}$ (25\% exact exchange), M06$2 \mathrm{X}^{62}$ (54\% exact exchange) and the range-separated hybrid CAM-B3LYP. ${ }^{63}$ The HOMO and the LUMO are always $\pi$ and the $\pi^{*}$ states, respectively, as shown in the projecteddensity of states (pDOS) plotted in Fig. S1, except for PBE, for which the $\mathrm{O} 2 \mathrm{p}$ states are at the edge of the band gap. PBE predicts a small KS gap of $3.58 \mathrm{eV}$ consistent with previous studies, ${ }^{28}$ while PBE0, M06-2X and CAM-B3LYP predictions range from $5.63 \mathrm{eV}$ to $7.83 \mathrm{eV}$. The $G W$ quasi- 
particle gap shows resilience to variations in the starting $\mathrm{XC}$ functional and the associated input Kohn-Sham eigenstates, and yields values ranging from $7.81 \mathrm{eV}$ and $7.95 \mathrm{eV}$ (gas phase: 9.23 and $9.37 \mathrm{eV}$ ). The computed values for the KS gap and the $G W$ gap are reported in Tab. 1. The renormalization of the fundamental gap of BDC due to dielectric solvent effects is large, i.e. $\sim 1.4 \mathrm{eV}$. On the other hand the optical gap is negligibly affected $(\sim 0.1 \mathrm{eV})$ consistent with a reduced effect of the polarizable medium on neutral excitations. The BSE spectra corresponding to different functionals are reported in Fig. S3. The small dependence of the $G W$ and BSE results on the XC choice confirms the robustness of the method. A further validation of the approach is provided by the good resemblance between the computed quasiparticle gap $(9.3 \mathrm{eV})$ and optical gap $(4.6 \mathrm{eV})$ of gas phase BDC ligand and the experimental values reported for the benzene molecule $(10.4 \mathrm{eV}$ and $4.8 \mathrm{eV}) .{ }^{64,65}$

For the periodic MOF-5, the total DOS and the DOS projected onto the $\mathrm{C}-2 \mathrm{p}$ of the ligand, the $\mathrm{O}-2 \mathrm{p}$ of the carboxylate group and the $\mathrm{Zn}_{4} \mathrm{O}$ metal node for PBE and GauPBE are plotted in Fig. 2. As expected, and in agreement with previous studies on the same MOF, ${ }^{9,28,29,66-68}$ the use of a hybrid functional leads to a gap opening by $\sim 1 \mathrm{eV}$ $(4.48 \mathrm{eV})$ with respect to PBE $(3.57 \mathrm{eV})$. PBE places the states localized on $\mathrm{O}-2 \mathrm{p}$ and $\mathrm{Zn}_{4} \mathrm{O}$ very close to the band gap while for Gau-PBE the frontier states are localized on the BDC ligand, with little contribution from the carboxylate $\mathrm{O}-2 \mathrm{p}$, similar to the ligand case discussed above. In $G W$, the HOMO-LUMO gap is widened greatly to $8 \mathrm{eV}$ and the pDOS undergoes a rearrangement as shown in Fig. 2: the frontier states $\left(v_{1}, v_{2}, c_{1}\right)$ are localized on the ligands and the deeper occupied and unoccupied states $\left(v_{3}, c_{2}\right)$ are localized on the zinc-oxide node. The $G W / \mathrm{PBE}$ band gap is $8.12 \mathrm{eV}$, close to the $G W / \mathrm{Gau}-\mathrm{PBE}$ value of $7.97 \mathrm{eV}$.

To gain further insight into the neutral and optical excitations in MOF-5, we compute $G W$ and BSE using two fragment models for the MOF. These are illustrated in Fig. S2. The corresponding Kohn-Sham and $G W$ gaps are tabulated in Tab. 1. As expected, the $G W$ band gap of both fragments without dielectric embedding is slightly overestimated: we compute $8.75 \mathrm{eV}$ for the $G W / \mathrm{PBE} 0$ gap of the bigger fragment (B) composed of four metal nodes and three ligand (7.97 $\mathrm{eV}$ for the periodic MOF using $G W / \mathrm{Gau}-\mathrm{PBE})$. The correction due to environment decreases the band gap by only $0.4 \mathrm{eV}$, confirming the small dielectric screening by the MOF environment. The reasonable good agreement between the fragment model upon inclusion of the environmental screening and the periodic calculations (within less than $0.3-$ $0.4 \mathrm{eV}$ ) suggest that molecular fragments can be employed to describe charged excitations in these MOFs. Even the small fragment (A) which includes only one ligand (see Fig. S2) and two metal nodes can provide a reasonably good description of the quasiparticle gap.

The BSE/ $G W /$ Gau-PBE optical spectrum of the periodic MOF-5 is reported in Fig. 3 together with the experimental one reported very recently. ${ }^{43}$ An excellent agreement between theory and experiment is found. The excitations are shown with red, orange and violet dots and the hole-electron densities corresponding to these excitations are visualized in lower panels of Fig. 3. These show a good resemblance with the ligand case (see Fig. 1) except for some minor localization on the zinc-oxide metal node. In Tab. 2 we report the composition of these excitations in terms of the occupied $(v)$ and unoccupied $(c)$ states $\left(v_{1}, v_{2}, v_{3}, c_{1}\right.$, and $\left.c_{2}\right)$ plotted in
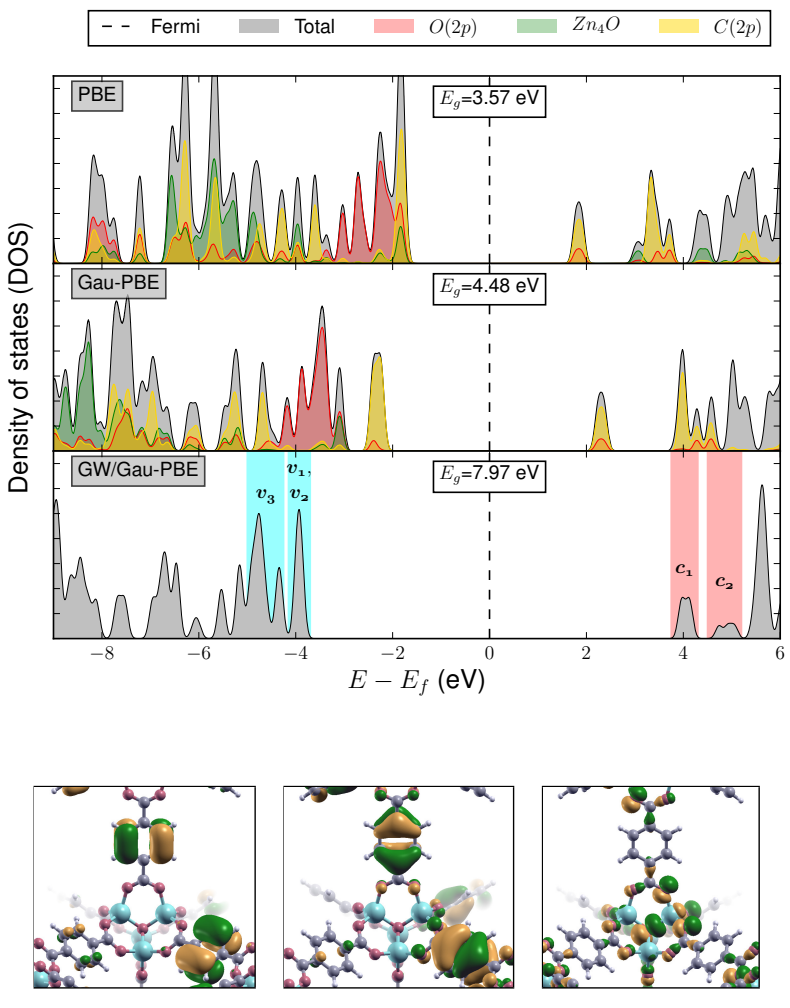

$v_{2}$

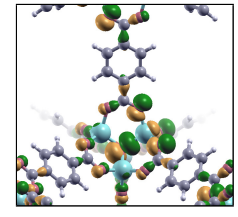

$v_{3}$

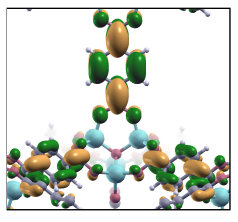

$c_{1}$

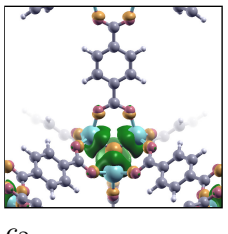

$c_{2}$

Figure 2. Density of states of periodic MOF-5 using PBE, Gau$\mathrm{PBE}$ and $e v G W / \mathrm{Gau}-\mathrm{PBE}$ approaches (upper figure). KohmSham eigenstates for the valence $(v)$ and conduction $(c)$ states highlighted in the DOS (lower figure).

Fig. 2.

The first weak excitation located at $4.5 \mathrm{eV}$ (in red) is mostly ligand-centered (68\% composed by $\pi \rightarrow \pi^{*}$ or $v_{1} \rightarrow$ $\left.c_{1}\right)$ and to a lesser extent ligand-to-metal $\left(26 \%, v_{2} \rightarrow c_{2}\right)$. The next intense band is composed by two groups of excitations close in energy but with different character. The excitations marked with orange $(5.21 \mathrm{eV})$ are mainly composed of transition from the metal-oxide node and carboxylate $\mathrm{O}$ atoms to the benzene ring $\left(v_{2} \rightarrow c_{1}\right)$. For the third set (violet), the most intense excitations are composed of ligand-centered $\pi \rightarrow \pi^{*}$ transitions (70\%) with a small contribution from metal-node $\left(v_{3} \rightarrow c_{2}, 12 \%\right)$. A good agreement is found between the spectra of the periodic MOF-5 computed using BSE/GW/Gau-PBE and BSE/GW/PBE as shown in Fig. S6. Also, the BSE/GW/PBE0 spectrum of the molecular model $\mathrm{B}$ is in very good agreement with the BSE $/ G W /$ Gau-PBE of the periodic MOF-5 (see Fig. S7) owing to the localized character of the excitons. The difference between the quasiparticle gap and the optical band gap (see Tab. 1) represents the binding energy of the hole and electron of the corresponding exciton which is $3.5 \mathrm{eV}$ for MOF-5 (Gau-PBE) and $3.3 \mathrm{eV}$ for the BDC ligand (PBE0) for the first excitation. 
Table 1. DFT gap, quasiparticle gap and optical gap, all in eV, of MOF-5 and BDC ligand calculated using different computational approaches. Values in parenthesis correspond to calculations using the dielectric embedding scheme discussed in the text. These should be used when comparing the results of the fragment models with the periodic MOF-5.

\begin{tabular}{ccccc}
\hline \hline & System & \multicolumn{2}{c}{ Energy gap (eV) } & Optical gap (eV) \\
\hline DFT & Model & Kohn-Sham & evGW & BSE \\
\hline PBE & ligand & $3.59(3.74)$ & $9.23(7.81)$ & $4.57(4.48)$ \\
M06L & ligand & $4.07(4.07)$ & $9.37(7.95)$ & $4.63(4.46)$ \\
PBE0 & ligand & $5.63(5.57)$ & $9.30(7.87)$ & $4.61(4.53)$ \\
M06-2X & ligand & $7.55(7.48)$ & $9.38(7.91)$ & $4.66(4.56)$ \\
CAM-B3LYP & ligand & $7.87(7.80)$ & $9.33(7.88)$ & $4.65(4.56)$ \\
\hline PBE & MOF-5 (periodic) & 3.57 & 8.12 & 4.58 \\
Gau-PBE & MOF-5 (periodic) & 4.48 & 7.97 & 4.49 \\
PBE0 & MOF-5 (frag. A) & 5.37 & $8.62(8.23)$ & $4.52(4.53)$ \\
PBE0 & MOF-5 (frag. B) & 5.32 & $8.75(8.37)$ & $4.80(4.80)$ \\
\hline \hline
\end{tabular}

Table 2. Composition of the discussed excitations computed using BSE/GW/Gau-PBE for the periodic MOF-5.

\begin{tabular}{ccc}
\hline Energy & Osc. Str. & Composition \\
\hline 4.49 (red) & 0.002 & $v_{1} \rightarrow c_{1}(68 \%), v_{2} \rightarrow c_{2}(26 \%)$ \\
5.21 (orange) & 0.017 & $v_{2} \rightarrow c_{1}(7 \%), v_{3} \rightarrow c_{1}(67 \%)$ \\
5.30 (pink) & 1.85 & $v_{2} \rightarrow c_{1}(70 \%), v_{3} \rightarrow c_{1}(6 \%), v_{1} \rightarrow c_{2}(1 \%), v_{3} \rightarrow c_{2}(12 \%)$ \\
\hline
\end{tabular}

We note that if one neglects the lowest energy peak in the UV-Vis absorption spectra reported by the early study by Tachikawa et al. ${ }^{18}$ which was later attributed to the $\mathrm{ZnO}$ impurity (at $3.6 \mathrm{eV}$ ), the agreement with our results is excellent. In their work, the most intense band is located at $\sim 5.2$ $\mathrm{eV}(240 \mathrm{~nm})$ and the next one with much lower intensity is found at $4.3 \mathrm{eV}(290 \mathrm{~nm})$, in agreement with our study and with the recent experimental reports by Rathnayake et al. ${ }^{43}$

\section{Conclusion}

In summary, we show that the well known severe underestimation of the fundamental gap by PBE is confirmed also in the case of MOF-5. While the PBE gap differs substantially from the fundamental gap, it gives a more reasonable estimate of the first excitation energy in agreement with the analysis reported in Refs. 69,70. A quantitatively good description of the optical gap and the optical absorption spectrum (compared with experiment) is obtained using the BSE/ $G W$ method. Unlike a recent TD-DFT study showing similar values for the fundamental and the optical gap for a series of MOFs, ${ }^{71}$ we show a large difference of more than $3 \mathrm{eV}$ with strong implications in the design of MOFs for optoelectronics ${ }^{33}$ or photocatalytic $\mathrm{CO}_{2}$ reduction. ${ }^{72,73}$ The insulating character of MOF-5 is not surprising considering that the frontier states are localized on the ligand molecules with small mixing with the $\mathrm{Zn}$ nodes. The highly localized nature of the exciton coupled with the low screening by the MOF environment yield strongly bound electron-hole pairs with binding energies of several eVs. The almost $50 \%$ difference between the fundamental and the optical gap indicates that the use of UV-Vis spectroscopy for the determination of fundamental band gaps in complex hybrid materials such as MOFs is highly questionable. Direct and inverse photoemission spectroscopy should be employed to this purpose and we are confident that experiments of this kind will confirm our results.

Acknowledgement This work benefited from the support of the project ANR-15-CE06-0003-01 funded by the
French National Agency for Research. Calculations were performed using resources granted by GENCI under the CINES grant number A0020907211. Additionally, the froggy platform of the CIMENT infrastructure, which is supported by the Rhone-Alpes region (GRANT CPER07_13 CIRA) and the Equip@Meso project was employed for the calculations.

\section{References}

(1) Qian, Q.; Asinger, P. A.; Lee, M. J.; Han, G.; Rodriguez, K. M.; Lin, S.; Benedetti, F. M. Wu, A. X.; Chi, W. S.; Smith, Z. P. MOF-Based Membranes for Gas Separations. Chemical Reviews 2020, 120, 8161-8266.

(2) Ding, M.; Flaig, R. W.; Jiang, H.-L.; Yaghi, O. M. Carbon capture and conversion using metal-organic frameworks and MOFbased materials. Chem. Soc. Rev. 2019, 48, 2783-2828.

(3) $\mathrm{Hu}$, Z.; Wang, Y.; Shah, B. B.; Zhao, D. $\mathrm{CO}_{2}$ Capture in MetalOrganic Framework Adsorbents: An Engineering Perspective. Advanced Sustainable Systems 2019, 3, 1800080.

(4) Resines-Urien, E.; Burzurí, E.; Fernandez-Bartolome, E.; García García-Tuñón, M. Á.; de la Presa, P.; Poloni, R.; Teat, S. J.; Costa, J. S. A switchable iron-based coordination polymer toward reversible acetonitrile electro-optical readout. Chem. Sci. 2019, 10, 6612-6616.

(5) He, H.; Cui, Y.; Li, H.; Shao, K.; Chen, B.; Qian, G. Controllable broadband multicolour single-mode polarized laser in a dye-assembled homoepitaxial MOF microcrystal. Light: Science \& Applications 2020,9.

(6) Gamonal, A.; Sun, C.; Mariano, A. L.; FernandezBartolome, E.; Guerrero-SanVicente, E.; Vlaisavljevich, B.; Castells-Gil, J.; Marti-Gastaldo, C.; Poloni, R.; Wannemacher, R.; Cabanillas-Gonzalez, J.; Costa, J. S. Divergent Adsorption-Dependent Luminescence of Amino-Functionalized Lanthanide Metal-Organic Frameworks for Highly Sensitive NO2 Sensors. The Journal of Physical Chemistry Letters $\mathbf{2 0 2 0}, \mathbf{1 1}, 3362-3368$.

(7) Li, H.; Eddaoudi, M.; O'Keeffe, M.; Yaghi, O. M. Design and synthesis of an exceptionally stable and highly porous metalorganic framework. Nature 1999, 402, 276-279.

(8) Stock, N.; Biswas, S. Synthesis of Metal-Organic Frameworks (MOFs): Routes to Various MOF Topologies, Morphologies, and Composites. Chemical Reviews 2012, 112, 933-969, PMID: 22098087.

(9) Butler, K. T.; Hendon, C. H.; Walsh, A. Electronic Structure Modulation of Metal-Organic Frameworks for Hybrid Devices. ACS Applied Materials \& Interfaces 2014, 6, 22044-22050.

(10) Alvaro, M.; Carbonell, E.; Ferrer, B.; Llabrés i Xamena, F.; Garcia, H. Semiconductor Behavior of a Metal-Organic Framework (MOF). Chemistry - A European Journal 2007, 13, 5106-5112.

(11) Rosi, N. L.; Eckert, J.; Eddaoudi, M.; Vodak, D. T.; Kim, J.; O'Keeffe, M.; Yaghi, O. M. Hydrogen Storage in Microporous Metal-Organic Frameworks. Science 2003, 300, 1127-1129.

(12) Saha, D.; Bao, Z.; Jia, F.; Deng, S. Adsorption of $\mathrm{CO}_{2}, \mathrm{CH}_{4}$ $\mathrm{N}_{2} \mathrm{O}$, and $\mathrm{N}_{2}$ on MOF-5, MOF-177, and Zeolite 5A. Environmental Science \&f Technology 2010, 44, 1820-1826. 

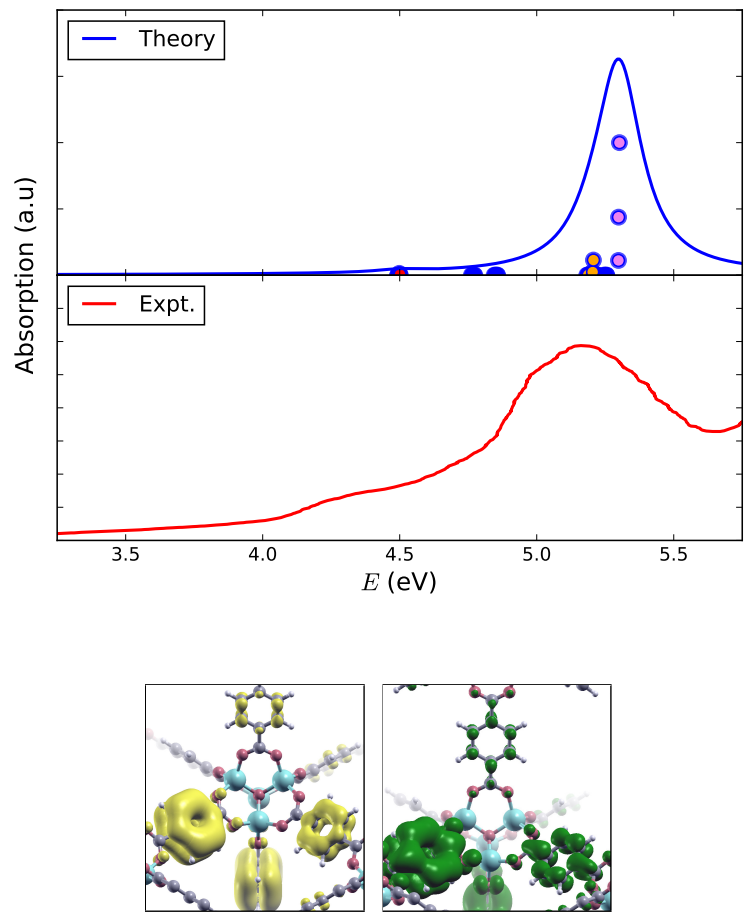

Hole

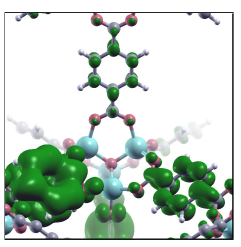

Electron

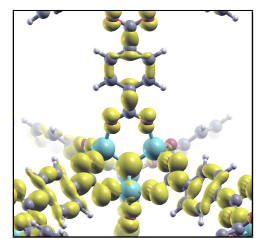

Hole

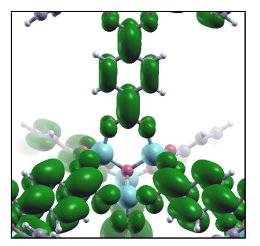

Electron

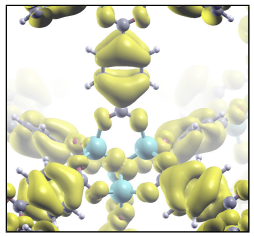

Hole

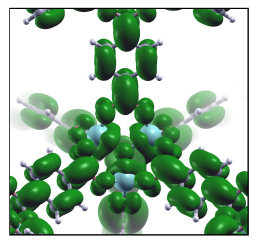

Electron

Figure 3. Optical absorption spectrum of MOF-5 computed using $\mathrm{BSE} / \mathrm{e} v G W / \mathrm{Gau}-\mathrm{PBE}$ (upper panel) and measured (lower panel) by Rathnayake et al. ${ }^{43}$ Hole and electron densities of the excitons reported as colored dots in the spectrum (lower figure).

(13) He, L.; Liu, Y.; Liu, J.; Xiong, Y.; Zheng, J.; Liu, Y.; Tang, Z. Core-Shell Noble-Metal@Metal-Organic-Framework Nanoparticles with Highly Selective Sensing Property. Angewandte Chemie International Edition 2013, 52, 3741-3745.

(14) Song, J.; Zhang, Z.; Hu, S.; Wu, T.; Jiang, T.; Han, B. MOF$5 / \mathrm{n}-\mathrm{Bu} 4 \mathrm{NBr}$ : an efficient catalyst system for the synthesis of cyclic carbonates from epoxides and $\mathrm{CO} 2$ under mild conditions. Green Chemistry 2009, 11, 1031.

(15) Hermes, S.; Schröter, M.-K.: Schmid, R.; Khodeir, L.; Muhler, M.; Tissler, A.; Fischer, R. W.; Fischer, R. A. Metal@MOF: Loading of Highly Porous Coordination Polymers Host Lattices by Metal Organic Chemical Vapor Deposition. Angewandte Chemie International Edition 2005, 44, 6237-6241.

(16) Rodríguez, N. A.; Parra, R.; Grela, M. A. Structural characterization, optical properties and photocatalytic activity of MOF-5 and its hydrolysis products: implications on their excitation mechanism. RSC Advances 2015, 5, 73112-73118.

(17) Bordiga, S.; Lamberti, C.; Ricchiardi, G.; Regli, L.; Bonino, F.; Damin, A.; Lillerud, K.-P.; Bjorgen, M.; Zecchina, A. Electronic and vibrational properties of a MOF-5 metal-organic framework: $\mathrm{ZnO}$ quantum dot behaviour. Chem. Commun. 2004, $2300-2301$.

(18) Tachikawa, T.; Choi, J. R.; Fujitsuka, M.; Majima, T. Photoinduced Charge-Transfer Processes on MOF-5 Nanoparticles:
Elucidating Differences between Metal-Organic Frameworks and Semiconductor Metal Oxides. The Journal of Physical Chemistry $C$ 2008, 112, 14090-14101.

(19) Feng, P. L.; Perry, J. J.; Nikodemski, S.; Jacobs, B. W. Meek, S. T.; Allendorf, M. D. Assessing the Purity of MetalOrganic Frameworks Using Photoluminescence: MOF-5, $\mathrm{ZnO}$ Quantum Dots, and Framework Decomposition. Journal of the American Chemical Society 2010, 132, 15487-15489.

(20) Villemot, V.; Hamel, M.; Pansu, R. B.; Leray, I.; Bertrand, G. H. V. Unravelling the true MOF-5 luminescence. RSC Advances 2020, 10, 18418-18422.

(21) Rodríguez, N. A · Parra, R · Román E. S · Grela, M. A A simple computational model for MOF-5W absorption and photoluminescence to distinguish MOF-5 from its hydrolysis products. Journal of Materials Science 2020, 55, 6588-6597.

(22) Gascon, J.; Hernández-Alonso, M.; Almeida, A.; van Klink, G. Kapteijn, F.; Mul, G. Isoreticular MOFs as Efficient Photocatalysts with Tunable Band Gap: An Operando FTIR Study of the Photoinduced Oxidation of Propylene. ChemSus Chem 2008, 1, 981-983.

(23) Khajavi, H.; Gascon, J.; Schins, J. M.; Siebbeles, L. D. A.; Kapteijn, F. Unraveling the Optoelectronic and Photochemical Behavior of Zn4O-Based Metal Organic Frameworks. The Jour nal of Physical Chemistry $C$ 2011, 115, 12487-12493.

(24) Feldblyum, J. I.; Keenan, E. A.; Matzger, A. J.; Maldonado, S. Photoresponse Characteristics of Archetypal Metal-Organic Frameworks. The Journal of Physical Chemistry C 2012, 116, 3112-3121.

(25) Lin, L.-C.; Berger, A. H.; Martin, R. L.; Kim, J.; Swisher, J. A. Jariwala, K.; Rycroft, C. H.; Bhown, A. S.; Deem, M. W.; Haranczyk, M.; Smit, B. In silico screening of carbon-capture materials. Nature Materials 2012, 11, 633-641.

(26) Choi, J. H.; Choi, Y. J.; Lee, J. W.; Shin, W. H.; Kang, J. K. Tunability of electronic band gaps from semiconducting to metallic states via tailoring $\mathrm{Zn}$ ions in MOFs with Co ions. Phys. Chem. Chem. Phys. 2009, 11, 628-631.

(27) Yang, L.-M.; Vajeeston, P.; Ravindran, P.; Fjellvag, H. Tilset, M. Theoretical Investigations on the Chemical Bonding, Electronic Structure, And Optical Properties of the MetalOrganic Framework MOF-5. Inorganic Chemistry 2010, 49 10283-10290.

(28) Pham, H. Q.; Mai, T.; Pham-Tran, N.-N.; Kawazoe, Y.; Mizuseki, H.; Nguyen-Manh, D. Engineering of Band Gap in Metal-Organic Frameworks by Functionalizing Organic Linker: A Systematic Density Functional Theory Investigation. The Journal of Physical Chemistry C 2014, 118, 4567-4577.

(29) Warmbier, R.; Quandt, A.; Seifert, G. Dielectric Properties of Selected Metal-Organic Frameworks. The Journal of Physical Chemistry $C$ 2014, 118, 11799-11805.

(30) Perdew, J. P.; Levy, M. Physical Content of the Exact KohnSham Orbital Energies: Band Gaps and Derivative Discontinuities. Phys. Rev. Lett. 1983, 51, 1884-1887.

(31) Odoh, S. O.; Cramer, C. J.; Truhlar, D. G.; Gagliardi, L. Quantum-Chemical Characterization of the Properties and Reactivities of Metal-Organic Frameworks. Chemical Reviews 2015, 115, 6051-6111.

(32) Mancuso, J. L.; Mroz, A. M.; Le, K. N.; Hendon, C. H. Electronic Structure Modeling of Metal-Organic Frameworks. Chemical Reviews 2020, 120, 8641-8715.

(33) Stavila, V.; Talin, A. A.; Allendorf, M. D. MOF-based electronic and opto-electronic devices. Chem. Soc. Rev. 2014, 43, 59946010 .

(34) Hedin, L. New Method for Calculating the One-Particle Green's Function with Application to the Electron-Gas Problem. Phys. Rev. 1965, 139, A796-A823.

(35) Salpeter, E. E.; Bethe, H. A. A Relativistic Equation for BoundState Problems. Phys. Rev. 1951, 84, 1232-1242.

(36) Strinati, G. Application of the Green's Functions Methods to the Study of the Optical Properties of Semiconductors. Riv. Nuovo Cim. 1988, 11, 1-86.

(37) Onida, G.; Reining, L.; Rubio, A. Electronic excitations: density-functional versus many-body Green's-function approaches. Reviews of Modern Physics 2002, 74, 601-659.

(38) Sottile, F.; Marsili, M.; Olevano, V.; Reining, L. Efficient Ab Initio Calculations of Bound and Continuum Excitons in the Absorption Spectra of Semiconductors and Insulators. Phys. Rev. B 2007, 76, 161103.

(39) Ping, Y.; Rocca, D.; Galli, G. Electronic excitations in light absorbers for photoelectrochemical energy conversion: first principles calculations based on many body perturbation theory. Chem. Soc. Rev. 2013, 42, 2437-2469.

(40) Loos, P.-F.; Scemama, A.; Duchemin, I.; Jacquemin, D. Blase, X. Pros and Cons of the BetheâĂSSalpeter Formalism for Ground-State Energies. The Journal of Physical Chemistry Letters 2020, 11, 3536-3545, PMID: 32298578.

(41) Palummo, M.; Raimondo, L.; Hogan, C.; Goletti, C.; Trabattoni, S.; Sassella, A. Nature of Optical Excitations in Porphyrin Crystals: A Joint Experimental and Theoretical Study. The Journal of Physical Chemistry Letters 2021, 12, 869-875, PMID: 33428409.

(42) Golze, D.; Dvorak, M.; Rinke, P. The GW Compendium: A Practical Guide to Theoretical Photoemission Spectroscopy. Frontiers in Chemistry 2019, 7. 
(43) Rathnayake, H.; Saha, S.; Dawood, S.; Loeffler, S.; Starobin, J. Analytical Approach to Screen Semiconducting MOFs Using Bloch Mode Analysis and Spectroscopic Measurements. The Journal of Physical Chemistry Letters 2021, 884-891.

(44) Blase, X.; Attaccalite, C.; Olevano, V. First-Principles $G W$ calculations for fullerenes, porphyrins, phtalocyanine, and other Molecules of Interest for Organic Photovoltaic Applications. Phys. Rev. B 2011, 83, 115103.

(45) Kaplan, F.; Harding, M. E.; Seiler, C.; Weigend, F.; Evers, F.; van Setten, M. J. Quasi-Particle Self-Consistent GW for Molecules. J. Chem. Theory Comput. 2016, 12, 2528-2541.

(46) Giannozzi, P et al. QUANTUM ESPRESSO: a modular and open-source software project for quantum simulations of materials. Journal of Physics: Condensed Matter 2009, 21, 395502.

(47) Sangalli, D. et al. Many-body perturbation theory calculations using the yambo code. Journal of Physics: Condensed Matter 2019, 31, 325902.

(48) Hamann, D. R. Optimized norm-conserving Vanderbilt pseudopotentials. Physical Review $B$ 2013, 88 .

(49) Schlipf, M.; Gygi, F. Optimization algorithm for the generation of ONCV pseudopotentials. Computer Physics Communications 2015, 196, 36-44.

(50) Perdew, J. P.; Burke, K.; Ernzerhof, M. Generalized Gradient Approximation Made Simple. Physical Review Letters 1996, 7\%, 3865-3868.

(51) Song, J.-W.; Yamashita, K.; Hirao, K. Communication: A new hybrid exchange correlation functional for band-gap calculations using a short-range Gaussian attenuation (GaussianPerdue-Burke-Ernzerhof). The Journal of Chemical Physics 2011, 135, 071103.

(52) Aprà, E. et al. NWChem: Past, present, and future. The Journal of Chemical Physics 2020, 152, 184102.

(53) Neese, F. The ORCA Program System. WIREs Comput. Mol. Sci. 2012, 2, 73-78.

(54) Blase, X.; Attaccalite, C. Charge-Transfer Excitations in Molec ular Donor-Acceptor Complexes within the Many-Body BetheSalpeter Approach. Appl. Phys. Lett. 2011, 99, 171909.

(55) Duchemin, I. Jacquemin, D. Blase, X. Combining the GW formalism with the polarizable continuum model: A state-specific non-equilibrium approach. J. Chem. Phys. 2016, $144,164106$.

(56) Li, J.; D'Avino, G.; Duchemin, I.; Beljonne, D.; Blase, X. Combining the Many-Body GW Formalism with Classical Polarizable Models: Insights on the Electronic Structure of Molecular Solids. J. Phys. Chem. Lett. 2016, 7, 2814-2820.

(57) D'Avino, G.; Muccioli, L.; Zannoni, C.; Beljonne, D.; Soos, Z. G. Electronic Polarization in Organic Crystals: A Comparative Study of Induced Dipoles and Intramolecular Charge Redistribution Schemes. J. Chem. Theory Comput. 2014, 10, 49594971.

(58) Duchemin, I.; Guido, C. A.; Jacquemin, D.; Blase, X. The Bethe-Salpeter formalism with polarisable continuum embedding: reconciling linear-response and state-specific features. Chem. Sci. 2018, 9, 4430-4443.

(59) Kshirsagar, A. R.; D'Avino, G.; Blase, X.; Li, J.; Poloni, R. Accurate Prediction of the S1 Excitation Energy in Solvated Azobenzene Derivatives via Embedded Orbital-Tuned BetheSalpeter Calculations. Journal of Chemical Theory and Computation 2020, 16, 2021-2027.

(60) Vaiano, V.; Sarno, G.; Sacco, O.; Sannino, D. Degradation of terephthalic acid in a photocatalytic system able to work also at high pressure. Chemical Engineering Journal 2017, 312, 1019 .

61) Adamo, C.; Barone, V. Toward reliable density functional methods without adjustable parameters: The PBE0 model. J. Chem. Phys. 1999, 110, 6158-6170.

(62) Zhao, Y.; Truhlar, D. G. The M06 Suite of Density Functionals for Main Group Thermochemistry, Thermochemical Kinetics, Noncovalent Interactions, Excited States, and Transition Elements: Two New Functionals and Systematic Testing of Four M06-class Functionals and 12 other Functionals. Theor. Chem. Acc. 2008, 120, 215-241.

63) Yanai, T.; Tew, D. P.; Handy, N. C. A new hybrid exchangeâĂS correlation functional using the Coulomb-attenuating method (CAM-B3LYP). Chemical Physics Letters 2004, 393, 51 - 57.

(64) Modelli, A.; Distefano, G.; Guerra, M.; Jones, D. In Wavefunc tions and Mechanisms from Electron Scattering Processes; Gianturco, F. A., Stefani, G., Eds.; Springer Berlin Heidelberg: Berlin, Heidelberg, 1984; pp 19-23.

(65) Hiraya, A.; Shobatake, K. Direct absorption spectra of jetcooled benzene in 130-260 $\mathrm{nm}$. The Journal of Chemical Physics 1991, 94, 7700-7706.

(66) Civalleri, B.; Napoli, F.; Noël, Y.; Roetti, C.; Dovesi, R. Abinitio prediction of materials properties with CRYSTAL: MOF-5 as a case study. CrystEng Comm 2006, 8, 364-371.

(67) Butler, K. T.; Hendon, C. H.; Walsh, A. Electronic Chemical Potentials of Porous Metal-Organic Frameworks. Journal of the American Chemical Society 2014, 136, 2703-2706.

(68) Ryder, M. R.; Donà, L.; Vitillo, J. G.; Civalleri, B. Understanding and Controlling the Dielectric Response of Metal-Organic Frameworks. ChemPlusChem 2018, 83, 308-316.

(69) Baerends, E.; Gritsenko, O.; Van Meer, R. The Kohn-Sham gap, the fundamental gap and the optical gap: the physical meaning of occupied and virtual Kohn-Sham orbital energies. Phys. Chem. Chem. Phys. 2013, 15, 16408-16425.
(70) Baerends, E. J. From the Kohn-Sham band gap to the fundamental gap in solids. An integer electron approach. Physical Chemistry Chemical Physics 2017, 19, 15639-15656.

(71) Fumanal, M.; Corminboeuf, C.; Smit, B.; Tavernelli, I. Optical absorption properties of metal-organic frameworks: solid state versus molecular perspective. Phys. Chem. Chem. Phys. 2020 22, 19512-19521.

(72) Fu, Y.; Sun, D.; Chen, Y.; Huang, R.; Ding, Z.; Fu, X.; Li, Z. An Amine-Functionalized Titanium Metal-Organic Framework Photocatalyst with Visible-Light-Induced Activity for $\mathrm{CO}_{2} \mathrm{Re}-$ duction. Angewandte Chemie International Edition 2012, 51, 3364-3367.

(73) Fang, Z.-B.; Liu, T.-T.; Liu, J.; Jin, S.; Wu, X.-P.; Gong, X.-Q. Wang, K.; Yin, Q.; Liu, T.-F.; Cao, R.; Zhou, H.-C. Boosting Interfacial Charge-Transfer Kinetics for Efficient Overall $\mathrm{CO}_{2}$ Photoreduction via Rational Design of Coordination Spheres on Metal-Organic Frameworks. Journal of the American Chemical Society 2020, 142, 12515-12523, PMID: 32564596. 